\title{
Balancing Proliferation and Connectivity in PTEN-associated Autism Spectrum Disorder
}

\author{
Amanda K. Tilot ${ }^{1,5}$ • Thomas W. Frazier II ${ }^{1,2,6}$ • Charis Eng ${ }^{1,3,4,5,7,8}$
}

Published online: 28 April 2015

(C) The American Society for Experimental NeuroTherapeutics, Inc. 2015

\begin{abstract}
Germline mutations in PTEN, which encodes a widely expressed phosphatase, was mapped to 10q23 and identified as the susceptibility gene for Cowden syndrome, characterized by macrocephaly and high risks of breast, thyroid, and other cancers. The phenotypic spectrum of PTEN mutations expanded to include autism with macrocephaly only 10 years ago. Neurological studies of patients with PTENassociated autism spectrum disorder (ASD) show increases in cortical white matter and a distinctive cognitive profile, including delayed language development with poor working memory and processing speed. Once a germline PTEN mutation is found, and a diagnosis of phosphatase and tensin homolog (PTEN) hamartoma tumor syndrome made, the clinical outlook broadens to include higher lifetime risks for multiple cancers, beginning in childhood with thyroid cancer. First described as a tumor suppressor, PTEN is a major negative regulator of the phosphatidylinositol 3-kinase/protein kinase $\mathrm{B} /$ mammalian target of rapamycin (mTOR) signaling path-
\end{abstract}

Electronic supplementary material The online version of this article (doi:10.1007/s13311-015-0356-8) contains supplementary material, which is available to authorized users.

\section{Charis Eng}

engc@ccf.org

1 Genomic Medicine Institute, Lerner Research Institute, Cleveland Clinic, Cleveland, OH 44195, USA

2 Center for Autism, Pediatric Institute, Cleveland Clinic, Cleveland, OH 44195, USA

3 Taussig Cancer Institute, Cleveland Clinic, Cleveland, OH 44195, USA

4 Stanley Shalom Zielony Institute of Nursing Excellence, Cleveland Clinic, Cleveland, OH 44195, USA way - controlling growth, protein synthesis, and proliferation. This canonical function combines with less well-understood mechanisms to influence synaptic plasticity and neuronal cytoarchitecture. Several excellent mouse models of Pten loss or dysfunction link these neural functions to autism-like behavioral abnormalities, such as altered sociability, repetitive behaviors, and phenotypes like anxiety that are often associated with ASD in humans. These models also show the promise of mTOR inhibitors as therapeutic agents capable of reversing phenotypes ranging from overgrowth to low social behavior. Based on these findings, therapeutic options for patients with PTEN hamartoma tumor syndrome and ASD are coming into view, even as new discoveries in PTEN biology add complexity to our understanding of this master regulator.

Key Words PTEN hamartoma tumor syndrome · syndromic . genetics $\cdot$ social behavior $\cdot$ neuroimaging $\cdot$ mouse model

5 Department of Molecular Medicine, Cleveland Clinic Lerner College of Medicine, Cleveland, OH 44195, USA

6 Department of Pediatrics, Cleveland Clinic, Cleveland, OH 44195, USA

7 Department of Genetics and Genome Sciences, Case Western Reserve University, Cleveland, OH 44106, USA

8 Germline High Risk Focus Group, CASE Comprehensive Cancer Center, Case Western Reserve University, Cleveland, $\mathrm{OH} 44106$, USA 


\section{Introduction}

The PTEN tumor suppressor gene on $10 \mathrm{q} 23$ was originally identified as a cancer predisposition gene in 1996-7 [1-3]. Since then, prospective observational studies have demonstrated that those with germline PTEN mutations are at increased risk of breast (women), thyroid, renal, and colon cancers, as well as melanoma [4-6]. In the 10 years since germline mutations in PTEN were discovered as a cause of autism spectrum disorder (ASD) in children with macrocephaly, studies have proliferated examining their prevalence, as well as the roles of phosphatase and tensin homolog (PTEN) within the central nervous system (CNS) [7, 8]. Now validated as an ASD susceptibility gene, PTEN mutation testing is a major consideration in cases of ASD with macrocephaly [9]. As our understanding of the phosphatase's multiple roles in the CNS has progressed, so has our awareness of the lifetime health outlook for individuals carrying PTEN mutations. Today, while its role as a negative regulator of the phosphatidylinositol 3-kinase (PI3K)/protein kinase B (AKT)/mammalian target of rapamycin (mTOR) signaling pathway is well established, we are just beginning to appreciate the existence of an alternative isoform and new nuclear functions $[10,11]$. How these new facets of PTEN biology affect its activity in the CNS is still unknown. Today, many groups are focused on areas where there is duality in PTEN activity - either at the phenotypic level between cancerous and neurodevelopmental symptoms, or at the cellular level where PTEN simultaneously regulates proliferation and connectivity. Therapeutic approaches that replace PTEN functions, such as mTOR inhibition, show great promise in mouse models and are poised to become the next big advance in the care of patients with PTEN mutations.

\section{Connecting Phenotype and Genotype Across the Lifespan}

The first case study of a child with a PTEN mutation and autism (PTEN-ASD) described a boy who inherited a nonsense mutation from his mother, who herself was diagnosed with Cowden syndrome but did not have social or intellectual disabilities [12]. Following this report, which recommended $P T E N$ mutation screening in cases of macrocephaly with pervasive developmental delay, came the first estimate of mutation frequency in a prospective series of patients with macrocephaly and autism. In 2005, Butler et al. [13] reported 3 PTEN mutations in a series of 18 children with macrocephaly and ASD (Table 1). This benchmark prevalence of $17 \%$ remains near the weighted average reported across nearly 10 subsequent studies (Table 1$)[13,15-18,20,21]$. Together, these results provide a strong case for PTEN mutation testing in children with ASD and macrocephaly, and the utility of such endophenotypes within the general ASD population as guides for judicious use of genetic testing.

Macrocephaly is defined as an occipital-frontal circumference (OFC) $>2$ SDs from the mean for one's height, sex, and ethnicity. Several studies from the USA and Europe estimate the prevalence of macrocephaly within ASD to be around $20 \%[16,22]$. Macrocephaly within ASD is linked to increased brain mass and white matter volumes, local processing bias, and genetic syndromes like PTEN hamartoma tumor syndrome (PHTS) that have clinically actionable adult phenotypes $[16,23,24]$. Combined with ease of measurement, these associations make macrocephaly an important endophenotype within ASD, worthy of deeper study.

The degree of macrocephaly observed in patients with ASD and PTEN mutations is often more severe than that seen in those with wild-type PTEN, as measured by the number of SDs from the mean. For example, of the 6 PTEN mutationpositive patients reported in the study by Varga et al. [18] their OFCs ranged from 2.9 to 5.8 SDs above the mean. A 2011 study by our group at the Cleveland Clinic [25] examined OFCs in a cohort of 181 PTEN mutation-positive individuals, finding their average head size to be +3.5 SDs from average in adults and +5 SDs in the pediatric subset. While subgroup analyses were only performed for age, sex, and mutation type (missense, truncating, or whole-gene deletions), individuals could be enrolled in the study for PTEN mutation testing if they met 1 of several criteria, including the relaxed definition of Cowden syndrome or the presence of autism plus macrocephaly [25]. The degree of macrocephaly was above that reported in many other genetic syndromes, which may allow for a shorter differential diagnosis and more costefficient efforts to identify a causative mutation in children with extreme macrocephaly and ASD. In our experience, children with PTEN-ASD are frequently identified in the first 2 years of life, typically as a result of macrocephaly, at times so great that it necessitates delivery by cesarean section.

Neuroimaging studies are necessary to uncover the structural differences underlying such extreme macrocephaly, and bridge genotype with phenotype for patients with PTENASD. Two independent studies report structural magnetic resonance imaging (MRI) phenotypes in patients with PTENASD [26, 27]. The multinational cohort described by Vanderver et al. [27] was retrospectively selected based known white matter pathology $(n=23)$, and included patients with and without ASD. The study by Frazier et al. [26] was designed to identify phenotypic characteristics of PTEN-ASD compared with PTEN mutation-negative ASD patients with and without macrocephaly, as well as healthy controls. The former study focused on the periventricular white matter and perivascular spaces, finding that all patients had macrocephaly and multifocal white matter abnormalities, enlarged periventricular spaces, or both [27]. As a prospective study, we were able to measure volumes for total grey and white 
Table 1 PTEN mutation frequencies reported in 10 studies during the last decade

\begin{tabular}{lllll}
\hline Study [ref.] & Inclusion criteria & Approach & Screened (n) & Mutations, $n(\%)$ \\
\hline Marchese, 2014 [14] & ASD with macrocephaly & PTEN mutation analysis & 31 & $1(3)$ \\
Hobert et al., 2014 [15] & ASD & PTEN mutation analysis & 62 & $6(10)$ \\
Klein et al., 2013 [16] & ASD with macrocephaly & $P T E N$ mutation analysis & 23 & $5(22)$ \\
McBride et al., 2010 [17] & ASD & PTEN clinical sequencing & 39 & $2(5)$ \\
Varga et al., 2009 [18] & ASD & PTEN clinical sequencing & 60 & $5(8)$ \\
Orrico, 2009 [19] & Macrocephaly and a neurodevelopmental disorder & PTEN direct sequencing & 40 & $3(8)$ \\
Herman et al., 2007 [20] & ASD with macrocephaly & PTEN mutation analysis & 16 & $2(13)$ \\
Buxbaum et al., 2007 [21] & ASD with macrocephaly & PTEN direct sequencing, MLPA & 88 & $1(1)$ \\
Butler et al., 2005 [13] & ASD with macrocephaly & PTEN mutation analysis & 18 & $3(17)$ \\
Total & & & 387 & $28(7)$ \\
\hline
\end{tabular}

$\mathrm{ASD}=$ autism spectrum disorder; MLPA = multiplex ligation-dependent probe amplification

matter, multiple brain structures including the corpus callosum, as well as cortical thickness and white matter hypointensities [26].

In our cohort, structural brain MRI findings in PTEN-ASD were remarkably consistent across patients, and highlight the utility of studying a defined genetic subgroup when exploring the underlying brain pathophysiology of ASD [26]. Consistent with large head circumference, patients with PTEN-ASD had dramatically increased total brain size. However, megalencephaly was almost entirely driven by increased cortical white matter, including callosal volume, with grey matter increases being much less dramatic and cortical thickness attenuated relative to measurements in idiopathic macrocephalic and normocephalic ASD, as well as healthy controls [26]. The increases in white matter volume bear similarity to the OFC measurements of patients with PTEN-ASD, representing more extreme examples of what has been seen in idiopathic macrocephalic ASD $[24,26]$. The massively increased white matter was also grossly abnormal, as indicated by substantial elevations in white matter hypointense areas on T1 images. Hypointensities were seen throughout the deep white matter, had markedly elevated volume in all but one patient with PTEN-ASD, and these volumes decreased slightly at higher ages. Only the oldest patient with PTEN-ASD (age 25 years) in our cohort did not show an elevated volume of white matter hypointensities relative to idiopathic ASD and controls [26]. This pattern suggests abnormal development of myelination, possibly consistent with unraveling myelin in Pten mouse models, rather than progressive deterioration $[26,28,29]$. Diffusion tensor imaging studies are needed to characterize these white matter abnormalities carefully. Specifically, longitudinal evaluations of a larger cohort of patients with PTEN-ASD, including multimodal imaging, comprehensive neurobehavioral phenotyping, and molecular signaling, will be crucial in understanding the developmental nature of these findings and identifying the underlying biology driving these abnormalities.
Recently, a series of case reports demonstrated epileptic seizures in PTEN mutation-positive patients, often linked to cortical dysplasia [30-34]. In 2011, Conti et al. [34] presented the first case of a 14-year-old boy with a deletion of PTEN exon 2, whose seizures began when he was 8 years old and could be controlled with valproic acid. Soon afterward, 3 separate reports described PTEN mutation-positive patients with both cortical dysplasia and seizures. These cases are characteristic of PHTS in that their phenotypes are quite disparate, ranging from a patient with ASD whose seizures began as infantile spasms to a man who first presented with partial epilepsy at the age of 55 years and was found to have multiple skin lesions and colonic polyps [31,33]. It will take detailed phenotyping in larger cohorts to reveal the prevalence of epilepsy within PHTS, but these intriguing reports align well with the high rates of seizures in tuberous sclerosis complex (TSC), a separate condition also linked to the PI3K pathway.

While the physical manifestations of a PTEN mutation, such as extreme macrocephaly, guide mutation testing and diagnosis for patients with PTEN-ASD, we must understand their cognitive and behavioral profile in order to develop informed strategies for therapy or other interventions. In our large cohort of patients with well-phenotyped PTEN-ASD, an early history of fine and gross motor difficulties was ubiquitous. Almost all patients reported delays in independent walking accompanied by deviations from the usual pattern of rolling over, sitting up, crawling, and supported walking [26]. With age, fine motor deficits become more apparent in many individuals, requiring occupational therapy and accommodations. Abnormal language development, both receptively and expressively, is also common. Almost all patients ascertained to date have reported delays in first words and phrases, with a substantial minority (29\%) remaining nonverbal or minimally verbal after the age of 4 years. Verbal ability scores were also lower, with $59 \%$ of patients with PTENASD scoring $<60$ [26]. As with other idiopathic cases, a disproportionate reduction in expressive, but not receptive, 
language is common. As patients with PTEN-ASD reach childhood, cognitive deficits are readily discerned in the majority of patients, with intellectual disability (full-scale IQ $<70$ with functional impairment) occurring in $47 \%$ of patients and an additional $29 \%$ having borderline-to-low average intellectual functioning. Even after considering reductions in general intellectual ability, patients with PTEN-ASD show disproportionate reductions in working memory and processing speed relative to idiopathic ASD patients and healthy controls [26]. The reductions in processing speed are apparent even in brief interactions, with patients with PTEN-ASD having delayed response latencies to questions and directives. These cognitive weaknesses require accommodations for learning beyond typical intensive behavioral intervention methods, including using short, clear phrases when giving directives or asking questions, repeating phrases or asking attention questions to ensure information is received, and allowing for response delays. Intriguingly, not all patients with PTEN-ASD show obvious cognitive deficits, with a small proportion of our initial cohort (24\%) having average to slightly above average intellectual ability $[4,9,26]$. The lack of a detectable connection to mutation location or type and with significant observed variability across siblings with the same mutation, other molecular explanations, including background genetics or epigenetic mechanisms, merit exploration as determinants of cognitive phenotype. Further studies are also needed to compare the cognitive profile of PTEN-ASD with other syndromic forms of ASD, as well as other neurodevelopmental disorders. Such studies would help personalize behavioral therapy for patients with PTEN-ASD, and may add insight into how the neural functions of PTEN translate to regulation of human behavior.

Identification of a PTEN mutation allows for a molecular diagnosis of PHTS, irrespective of clinical diagnosis, and the beginning of a multispecialty cancer surveillance regimen that will continue through adulthood [35]. Cancer risks in individuals with PHTS, regardless of their ASD status, begin to diverge from the normal population in childhood. A thyroid ultrasound is recommended at the time of genetic diagnosis, as thyroid cancer can appear before the age of 10 years, and repeated annually [36]. With age, the risks for other solid tumors increase, and screening programs for breast, endometrial, and renal cancers are well reviewed elsewhere [4, 35]. Beginning at the age of 30-35 years, yearly cancer screenings should be expanded to include mammograms and/or breast MRI, as well as endometrial surveillance for women, colonoscopy, and a skin examination [4]. Kidney surveillance begins at the age of 40 years, continuing every other year [35]. Benign overgrowths associated with the syndrome include mucocutaneous lesions, facial trichilemmomas, acral keratosis, papillomatous papules, and mucosal lesions. These dermatologic features can be subtle but are highly predictive of a germline PTEN mutation [9].
There are several PTEN mutations seen in patients with and without ASD, beginning with the first case report in 2001 where the proband with autistic behaviors inherited a PTEN mutation from his neurotypical mother [12]. Apart from the extremely early risk of thyroid cancer, the cancer risks in PHTS typically begin to manifest in the patients' 30 s, and so a lack of cancer diagnoses in a child with ASD and PHTS is noninformative [4]. While the conclusion that non-PI3Krelated functions of PTEN may be critical factors for PHTSassociated ASD is important, there is no evidence yet to suggest that children with ASD and PTEN mutations are exempt from the cancer risks associated with the syndrome.

\section{PTEN Structure, Localization, and Signaling}

The spectrum of mutations seen in PHTS (with and without ASD) extends throughout the coding sequence of PTEN [9]. Located on human chromosome sub-band 10q23.3, the 9exon PTEN gene encodes a 403-amino acid phosphatase with four major domains $[37,38]$. Within the $\mathrm{N}$-terminal tail are several key motifs, including the phosphatidyl-inositolbisphosphate $\left(\mathrm{PIP}_{2}\right)$ binding motif and both nuclear and cytoplasmic localization sequences [11, 37, 39-41]. The protein phosphatase domain contains the catalytic core of the protein, stretching from amino acid 123 to 130 [37]. A C2 domain facilitates membrane binding, and the PDZ-binding motif within the C-terminal tail allows protein-protein interactions, such as those with the synaptic scaffolding protein postsynaptic density protein 95 [37, 42]. Recent reports identified a new isoform of PTEN generated by alternative splicing, named PTEN-long for its additional 173 amino acids at the Nterminus [43, 44]. Our cohort of 290 probands with PHTS revealed mutation hotspots in exons 5,7 , and 8 , together accounting for $61 \%$ of all pathogenic variations observed [9]. The exon 5 hotspot includes the catalytic core of PTEN, and mutations within this 7 -amino acid stretch affect probands with a wide variety of clinical presentations [9, 45]. Deletions and duplications affecting PTEN are less common within PHTS than single base pair alterations, though they can still be found over the entire coding sequence [9]. Even the largest cohorts of patients with PHTS are insufficient to identify clear genotype-phenotype associations, a limitation that prompted several attempts to cluster germline PTEN mutations by their impact on its phosphatase activity and signaling.

The best-studied function of PTEN is its plasma membrane-associated dephosporylation of $\mathrm{PIP}_{3}$ to $\mathrm{PIP}_{2}$, though this is far from its singular activity (Fig. 1) [46]. PIP 3 is produced by $\mathrm{PI} 3 \mathrm{~K}$ as a response to the binding of growth factors or other molecules to receptor tyrosine kinases, acting as a potent second messenger [47]. PTEN inhibition of PIP $_{3}$ results in decreased phosphorylation of the serine-threonine kinase AKT [46]. Elevation of this phosphorylation at 
Fig. 1 Neuronal PTEN signaling at the soma and synapse. $\mathrm{RTK}=$ receptor tyrosine kinase; IRS = insulin receptor substrate 1 ; RAS $=$ rat sarcoma; $\mathrm{RAF}=$ rapidly accelerated fibrosarcoma; MEK = mitogen-activated protein/ extracellular signal-regulated kinase kinase; ERK = extracellular regulated kinase; PI3K = phosphatidylinositol 3-kinase; $\mathrm{PIP}_{2}=$ phosphatidyl-inositolbiphosphate; $\mathrm{PIP}_{3}=$ phosphatidyl-inositoltriphosphate; PTEN = phosphatase and tensin homolog; AKT = protein kinase $\mathrm{B}$; $\mathrm{P}=$ phosphorylation; $\mathrm{TSC} 1=$ tuberous sclerosis complex 1 ; TSC2 = tuberous sclerosis complex $2 ;$ mTORC $1=$ mammalian target of rapamycin complex 1; 4EBP1 = eukaryotic translation initiation factor $4 \mathrm{E}$ binding protein $1 ;$ NMDAR $=\mathrm{N}$ methyl-D-aspartate receptor; PSD-95 = postsynaptic density protein 95

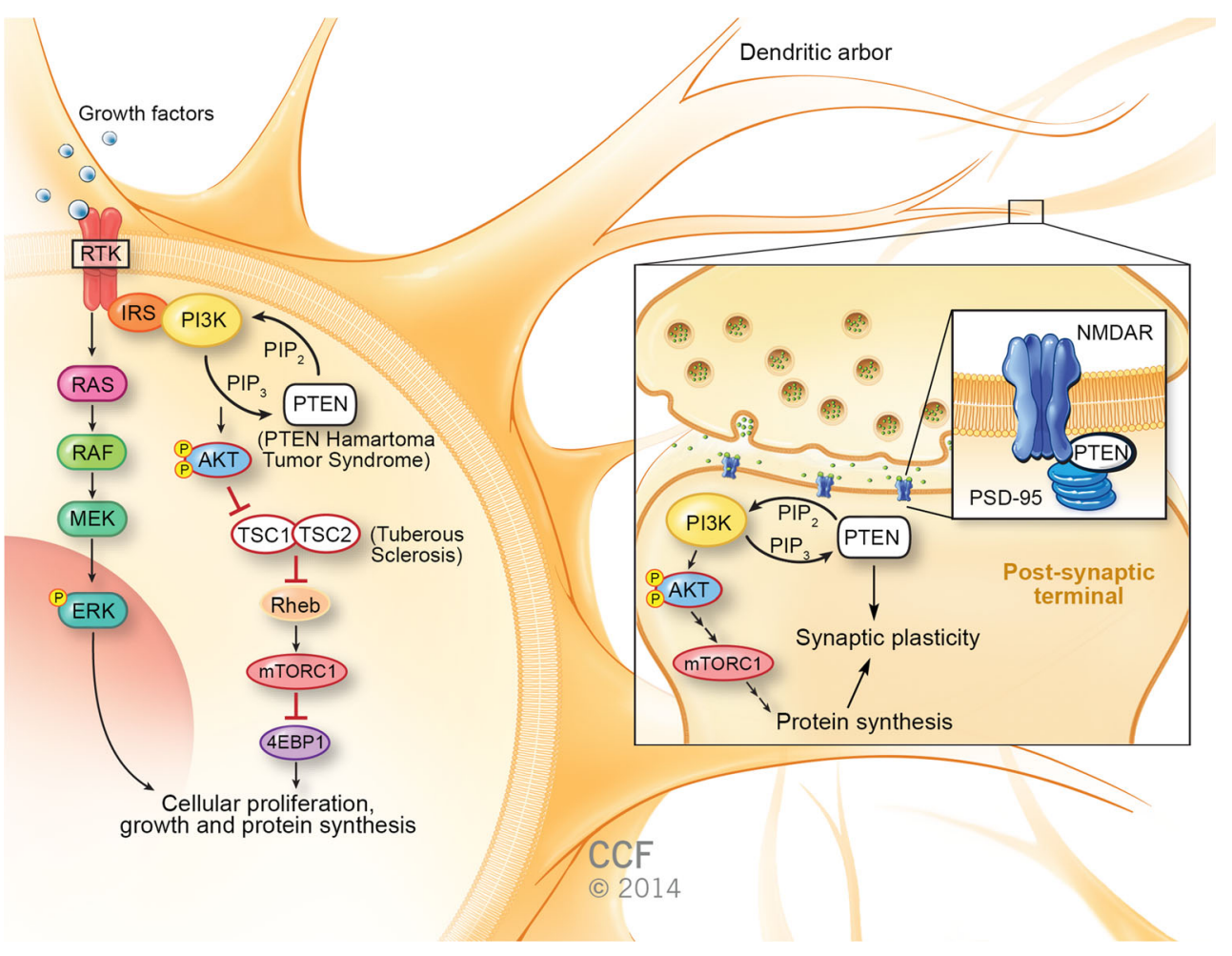

Ser473 and Thr308 are common readouts of PTEN loss. Utilizing lymphoblastoid cell lines (LCLs) generated from patients with PHTS, our group demonstrated that, on average, germline PTEN mutations lead to reductions in PTEN protein levels and increases in phosphorylated AKT1 [9]. In fact, there was a strong inverse association between a patient's phenotypic burden and the level of PTEN protein observed in their LCL. The AKT/mTOR signaling pathway is well studied within both the cancer and neuroscience communities. AKT alone is a major activator of several cellular processes, including protein synthesis, cell cycle progression, and survival. Activation of AKT allows it to phosphorylate TSC2, thus removing the TSC1/2 complex's inhibition of mTOR complex 1 [48]. In turn, mTOR activation promotes ribosome biogenesis and increased translation efficiency. Mutations in TSC1/2 underlie TSC, another genetic syndrome with high rates of ASD, and together with PTEN suggest that tight regulation of the PI3K/AKT/mTOR pathway is essential for normal social behavior. Indeed, clinical trials assessing the benefit of mTOR inhibitors for autism and other neuropsychological symptoms of TSC are underway, using compounds already approved for other indications (everolimus).

In a hunt for a distinguishing feature of germline PTEN mutations that lead to ASD compared with those only identified in patients without ASD, multiple groups have interrogated their effects on PTEN phosphatase activity and AKT inhibition. Reporting an H93R missense mutation observed in a child with ASD, Redfern et al. [49] found disrupted binding to $\mathrm{PIP}_{2}$, leading to increased association with the plasma membrane. This mutant PTEN also exhibits reduced lipid phosphatase activity, perhaps due to structural alterations that prevent the necessary conformational switch. Their results sparked the hypothesis that ASD-associated PTEN mutations affect AKT inhibition differently than those appearing in adults with predominantly cancer phenotypes. Using a yeast reconstitution system, one study measured the phosphatase activity of 14 mutations associated with ASD or developmental delay [50]. It found that the majority of these mutations retained most of their phosphatase activity, in contrast to the more severe functional impairment seen in a panel of PTEN mutations that were only reported in cases of PHTS without ASD [50]. These results were recently confirmed by Spinelli et al. [51] who reported better AKT inhibition in 7 PTEN mutations seen only in ASD with PHTS cases compared with 5 mutations observed in patients with PHTS with high tumor burdens.

While these reports are provocative, and would suggest lower cancer risks for patients with these ASD-linked mutations, studies from our group provide evidence that levels of AKT inhibition are unlikely to provide meaningful clinical distinction. From our cohort of nearly 300 PHTS probands plus their affected family members, we know that the phenotypic effects of germline PTEN mutations can be highly variable, even within a single mutation [45]. We found that pathogenic mutations, unselected for phenotype, led to increases in phosphorylated AKT (p-AKT) in cell lines derived from patient blood samples (LCLs) [9]. Combined with the clinical perspective that $P T E N$ mutations first identified in a pediatric 
setting are poor indicators of later tumorigenic potential, this result indicates that inhibition of PI3K signaling cannot predict risk of neurodevelopmental or cancer phenotypes. Supporting this assertion, we published a report describing a wide variety of clinical phenotypes in patients with identical missense or nonsense mutations [45]. In this same study, we divided PTEN mutation-positive patients into those with and without elevated proteasome activity, which was associated with lower PTEN protein levels. Neurological symptoms, including ASD, developmental delay, and intellectual disability, were more common amongst patients with high proteasome activity - even though their mutations were also found in patients without such symptoms [45]. Increases in the identification of ASD, including a massive increase in prevalence estimates from $\sim 5$ in 10,000 in the 1980s to 1 in 68 in 2014, have provided an additional challenge to comprehensive cohort ascertainment in studies of genetic forms of ASD, particularly over the last decade, in which awareness and ascertainment have changed most dramatically [52-55]. Future studies seeking a way to predict which patients with PHTS will develop neurodevelopmental phenotypes such as ASD should turn their attention to non-PI3K-related functions of PTEN, and consider dividing patient cell lines by their molecular phenotypes rather than specific mutations.

As we continue to discover noncanonical roles for PTEN, the importance of its intracellular localization to its activity becomes more pronounced. The activities of nuclear PTEN are numerous but remain poorly studied compared with its cytoplasmic roles. PTEN translocates to the nucleus via multiple mechanisms, including monoubiquitination and interaction with major vault protein [56, 57]. Its nuclear export appears dependent on active transport or association with Ran-guanosine triphosphatase [39, 58]. As in the cytoplasm, nuclear PTEN still exhibits lipid phosphatase activity, including inhibition of AKT phosphorylation [45]. In addition, studies show that nuclear PTEN can inhibit mitogenactivated protein kinase signaling, promote apoptosis independently of p53, and aid chromosomal stability through association with centromere protein C $[59,60]$. A very recent report indicates that nuclear PTEN also contributes to chromatin condensation, and thus transcriptional repression at a genome-wide level, through physical interactions with histone H1 [10]. Additional cytosolic functions of PTEN may be found in the activity of PTEN-long, as recent studies suggest that this isoform may aid adenosine triphosphate production within mitochondria [44]. That new isoforms and functions of PTEN are still being uncovered underscores how little we know about this major regulatory protein. Relating these discoveries to the neurological functions of PTEN, and PHTS as a whole, may bring new insights into how mutations in one protein can produce such varied clinical phenotypes.

\section{Pten Loss in Mice Leads to Cytoarchitecture and Synaptic Alterations}

Fifteen years of applying mouse models to the study of Pten neurobiology reveal a remarkably consistent set of cellular processes regulated by this phosphatase. The picture muddies somewhat in the details, recent examples being the directionality of Pten regulation or how phenotypes manifest in conditional knockouts (cKOs) compared with germline disruption models [61-65]. The 1998 discovery that homozygous deletion of Pten in mice causes embryonic lethality by embryonic day 7.5 directed many groups toward homozygous cKOs to investigate Pten functions in the CNS [66]. Pten heterozygous animals are viable, and along with the differentiated neuronrestricted model Nse-cre;Pten ${ }^{\text {loxP/loxP }}$, represent the best phenotyped models for Pten loss in the CNS [62, 66]. Extending findings from these earlier models, second generations of Pten cKOs with more restricted Pten loss were used to pinpoint the minimal cell types and time points required for normal behavioral and synaptic development $[67,68]$. While neuronal models have the most complete phenotyping for ASD-like deficits, the emergence of glial gene expression signatures in studies of neural tissue from individuals with ASD prompts further consideration of roles for PTEN in astrocytes and oligodendrocytes as well [69]. As we consider both overgrowth (macrocephaly, cancer) and neurodevelopment in PHTS, these models provide excellent tools for uncovering how PTEN simultaneously regulates proliferation and connectivity in the CNS.

Hyperproliferation is a nearly ubiquitous finding in models of neural Pten loss, usually a result of elevated AKT/mTOR signaling. The effect is particularly striking in progenitor populations, which must balance renewal of the stem population with the generation of fate-restricted daughter cells [67]. In the Nestin-CreER ${ }^{T 2}$ mouse, Pten loss in neural progenitors caused a burst of proliferation and generation of new neurons. This increase was followed by early terminal differentiation that was skewed toward the astrocytic lineage [67]. In the Pten $^{m 3 m 4}$ model, disruption of Pten localization toward cytoplasmic predominance led to a substantial increase in neural/ glial antigen 2-positive polydendrocyte proliferation accompanied by greater numbers of astrocytes and oligodendrocytes, without affecting the density of neurons or microglia [61]. As we learn more about the importance of the farreaching PI3K pathway in ASD pathogenesis, it is tempting to speculate that it may also be involved in the temporary elevation of early brain growth seen in some patients with ASD, which normalizes as neurotypical children catch up between the age of 6 and 10 years [70].

Increased cell size is another hallmark feature of lowered Pten activity, seen across cell types and modes of Pten disruption. That total Pten loss in neurons and astrocytes causes sufficient increases in AKT/mTOR signaling to generate 
cellular hypertrophy is unsurprising $[62,71]$. Phenotype reversal after rapamycin (a mTOR complex 1 inhibitor) administration confirms that the increased p-AKT seen in the hypertrophic cells drives this process [72]. Connecting these proofof-principle findings with clinical data, subtler models of Pten disruption still show cellular hypertrophy resulting from more modest elevations in p-AKT. For example, the $\mathrm{Pten}^{m 3 m 4}$ mouse exhibits a $30 \%$ drop in neural Pten and 10-25\% increases in cell body size across multiple neuronal populations [61]. Gross size increases may be less important to the synaptic and behavioral phenotypes of these models than disrupted cytoarchitecture at points of information transfer, such as dendritic spines and the myelin sheath.

The consequences of increased AKT/mTOR signaling in morphologically complex cells like neurons and oligodendrocytes extend to both the shape and number of their projections. These changes in axons, dendritic spines, and myelin ultrastructure are likely signs of and contributors to altered connectivity in mouse models of Pten loss in the CNS. Multiple models of neuronal Pten loss, covering different populations and brain regions, exhibit increased branching of the dendritic arbor, thickening of these processes, and even ectopic axonal projections [61, 62, 73, 74]. Using Golgi staining to visualize these neurons, several groups also reported increased spine density in various regions $[62,72,74,75]$. Normalized gross morphology and spine counts following rapamycin administration confirmed that these overgrowth phenotypes involve elevated mTOR signaling [72]. Later, Haws et al. [76] used more sensitive cell-filling techniques to demonstrate that while the number and size of mature, mushroom-shaped spines was increased, total spine counts did not increase after Pten knockdown in vivo. This important finding suggests that the main effect of Pten signaling in dendritic spines is related to their stabilization and maturity, rather than sheer number. Integrating this finding into our understanding of how PI3K signaling functions during synaptic activity will clarify whether changes in spine shape are an independent effect of Pten loss or a secondary effect of increased synaptic activity. Alterations to the myelin sheath, including increased myelination, disrupted nodes of Ranvier, and splitting of the myelin lamellae, are major phenotypes in oligodendrocyterestricted models of Pten loss or AKT activation [28, 29, 77]. Similarly to the increased callosal volumes observed in patients with PTEN-ASD, Pten ${ }^{m 3 m 4}$ mice also exhibit upregulation of myelination-related genes and corpus callosum thickening, suggesting that even modest Pten disruption is sufficient to affect the myelination process [26, 61]. As evidence of glial involvement accumulates in studies of idiopathic ASD, more studies are needed to determine how altered myelination after PTEN disruption affects social and other ASD-linked behaviors.

Multilevel interaction between PTEN and the molecular machinery of synaptic communication represent a direct link to a hallmark feature of ASD neuropathology, described at the level of neuroimaging, as well as transcriptomic studies $[69,78]$. The first report placing Pten at the synapse came in 2004, when Peradones et al. [79] described PTEN localization in dendrites and their spines during developmental periods of synaptic stabilization. Linking canonical functions of PTEN with synaptic activity, turnover of the $\mathrm{PIP}_{2} / \mathrm{PIP}_{3}$ pool is critical for neurotransmitter vesicle cycling and PI3K activity is essential for long-term potentiation (LTP) [80]. While $\mathrm{PIP}_{3}$ levels rise as a result of PI3K activity during both LTP and long-term depression (LTD), PTEN reduces $\mathrm{PIP}_{3}$ levels only during $N$-methyl-D-aspartate receptordependent LTD, acting as a switchboard distinguishing the 2 forms of synaptic plasticity [80]. Downstream, LTP requires new protein synthesis at the dendritic spine, an mTOR signaling-dependent process that can be activated by brainderived neurotrophic factor following Pten degradation [81]. PTEN also appears to be involved in neurotransmitter receptor trafficking, binding to both the $\mathrm{N}$-methyl-D-aspartate receptor subunit NR2A and the scaffolding postsynaptic density protein $95[42,82]$. How these physical associations with other synaptic proteins contribute to connectivity and behavior are open questions deserving deeper study, especially as nonphosphatase activities of PTEN are increasingly considered as part of the etiology of ASD within PHTS.

These varied roles for PTEN in the molecular machinery of synaptic plasticity add to the regulation of both short- and long-range connectivity in the brain. Alterations in each of these processes are linked to ASD pathogenesis, suggesting that PTEN-regulated pathways may be point of convergence in the broad etiologies of the disorder [78]. Several reports indicate that PTEN inhibition of PI3K is essential for LTD, with conflicting reports on its requirement in LTP $[42,63]$. This role in synaptic plasticity may be disconnected from PTEN regulation of cell size and morphology, as multiple reports indicate that functional deficits arise prior to neuronal hypertrophy [64, 65]. The baseline electrical activity of PTEN-null neurons also appears to be altered in favor of increased excitatory postsynaptic current, as measured in both the hippocampus and basolateral amygdala $[75,76]$. These reports demonstrate an altered balance of excitatory and inhibitory activity paralleling findings of local hyperconnectivity in human ASD. Xiong et al. [83] showed that Pten loss in neurons of the auditory cortex led to increased synaptic responses to both local inputs and long-range stimulus across the corpus callosum. These increased responses could be blocked with rapamycin administration, suggesting that mTOR signaling is required [83]. While long-range hypoconnectivity is more commonly reported in human ASD, it is possible that such circuits are sensitive to disruption in either direction, especially during critical points in neurodevelopment. 
Autism-like Behavioral Phenotypes in Mouse Models of Pten Loss or Disruption

Phenotyping mouse models of both idiopathic and syndromic forms of ASD requires an appreciation for the range of behaviors seen in patients with autism and any abnormalities specific to the patient population of focus. There are several models of Pten disruption in mice that exhibit abnormal social behavior, usually in combination with other behavioral deficits (Table 2). Compared with wild-type mice, the first model to demonstrate reduced social behavior was the Nse-cre; Pten ${ }^{\text {loxP } / l o x P}$ mouse, which showed a reduced preference for another mouse than a nonsocial object [62]. This decrease in social behavior was replicated in later models based on Pten loss in more restricted populations, such as granule cells of the dentate gyrus and cerebellum or neural progenitors in adult animals [67, 87]. Having established the critical role of neuronal Pten activity for normal social behavior, other models add context for what may occur in PHTS where PTEN mutations are germline and behavioral phenotypes are the collective expression of this pathology in every cell type. Although their phenotypes are an imperfect match, the germline heterozygous $\mathrm{Pten}^{+/-}$mouse is the closest available model for patients with PHTS with PTEN alterations that lead to low protein dose, such as nonsense mutations or frameshift causing insertions/deletions [9]. Several studies give a thorough description of social and other ASD-linked behaviors in these animals [84, 85, 88], finding lower overall sociability and a number of sex-specific phenotypes. Most recently, our group identified inappropriately elevated social behavior in male $P_{t e n}{ }^{m 34}$ mice, where Pten localization is altered similarly to that seen in some PHTS missense mutations $[57,61]$. Together, these models point to an essential role of neuronal Pten in the regulation of social behavior, and hint that the molecular consequences of each specific mutation may influence the phenotypic outcomes. It is remarkable that differing alterations to the same gene can produce social behavior phenotypes that cover the full range of human ASD-from socially withdrawn children with intellectual disability (modeled well by Nse-cre; Pten ${ }^{\operatorname{loxP} / l o x P}$ mice) to the more socially motivated picture seen in high-functioning cases (perhaps modeled by Pten $^{m 3 m 4}$ mice) $[57,62]$.

In addition to social behavior deficits, several mouse models of Pten loss exhibit behaviors relevant to other core symptoms of ASD or frequent comorbidities. Repetitive behaviors, now a critical half of ASD diagnosis, are seen in Gfap-Cre; Pten ${ }^{\text {loxP/loxP }}$ and male $\mathrm{Pten}^{+/-}$animals as repetitive marble burying or selfgrooming [84, 87]. Although epilepsy is not part of

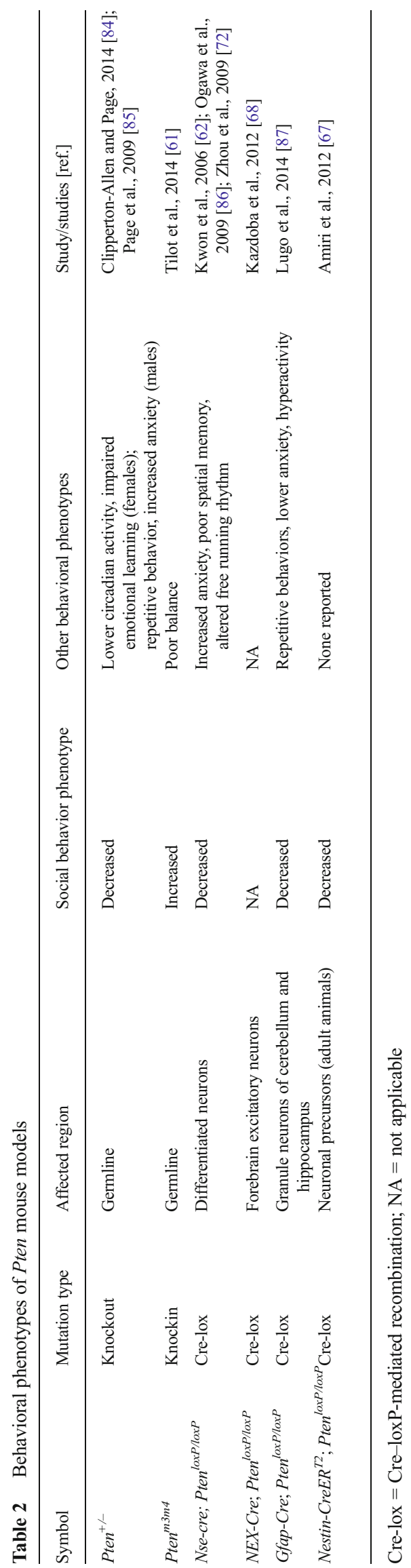


the diagnostic criteria for ASD, its association with germline PTEN mutations in several recent case reports adds new relevance to the seizure phenotypes observed in multiple mouse models of neural Pten loss [30-33, $62,86]$. Both increased anxiety and lack of appropriate fear are reported in human ASD, and this range of behaviors is also seen in models of neuronal Pten loss. The Gfap-Cre; Pten ${ }^{\text {loxP/loxP }}$ model exhibits lower than usual anxiety levels in combination with hyperactivity, while the Nse-cre; Pten ${ }^{\text {loxP/loxP }}$ mouse showed high levels of anxiety-like behavior, as well as increased locomotion in the open field test $[62,87]$. The Pten ${ }^{m 3 m 4}$ model also demonstrated poor balance on the accelerating rotarod task, potentially in keeping with the motor clumsiness reported in individuals with high-functioning ASD [61, 89]. Finally, altered circadian rhythms are noted in ASD, and are also reported in the Nse-cre; Pten ${ }^{\text {loxP/loxP }}$ model as disturbed free-running rhythm and lower overall circadian activity in female $\mathrm{Pten}^{+/}$ mice $[84,86]$.

\section{Conclusions}

Our understanding of PTEN as a major regulatory protein in both neurobiology and tumorigenesis has grown in parallel over the last decade. PHTS spans these 2 fields, as individuals bearing germline PTEN mutations must first face the risk of neurodevelopmental problems, followed by escalating risks for multiple cancers. Based on the work of many groups demonstrating the potential for mTOR inhibitors to prevent or even reverse phenotypes associated with Pten loss in mice, we await the results of a recent clinical trial that examined the effect of mTOR inhibition in adult patients with PHTS, with or without cancer (ClinicalTrials.gov identifier: NCT00971789). A third role of PTEN regulation in human biology is in metabolism, seen in patients with PHTS at high risk for insulin resistance and obesity [90]. As metabolic processes are foundational to both neurological function and tumorigenesis, targeting such imbalances in patients with PHTS may one day constitute an alternative to signaling-pathway based pharmacological interventions. It is highly probable that investigation of new avenues in PTEN biology will produce new targets or mechanisms amenable to therapeutic intervention, an important consideration as the number of noncanonical functions of PTEN continues to grow.

Acknowledgments Our human autism spectrum disorder-phosphatase and tensin homolog work is funded by National Institutes of Health grant 1U54NS092090-01 (to T.F. and C.E.), and generous donations from Sam and Maria Miller (to C.E.). C.E. is the Sondra J. and Stephen R. Hardis
Endowed Chair in Cancer Genomic Medicine at the Cleveland Clinic, and an American Cancer Society Clinical Research Professor.

Required Author Forms Disclosure forms provided by the authors are available with the online version of this article.

\section{References}

1. Liaw D, Marsh DJ, Li J, et al. Germline mutations of the PTEN gene in Cowden disease, an inherited breast and thyroid cancer syndrome. Nature Genet 1997;16:64-67.

2. Marsh DJ, Dahia PL, Zheng Z, et al. Germline mutations in PTEN are present in Bannayan-Zonana syndrome. Nature Genet 1997;16: 333-334.

3. Nelen MR, Padberg GW, Peeters EA, et al. Localization of the gene for Cowden disease to chromosome 10q22-23. Nature Genet 1996;13:114-116.

4. Tan MH, Mester JL, Ngeow J, Rybicki LA, Orloff MS, Eng C. Lifetime cancer risks in individuals with germline PTEN mutations. Clin Cancer Res 2012;18:400-407.

5. Guenard F, Labrie Y, Ouellette G, et al. Germline mutations in the breast cancer susceptibility gene PTEN are rare in high-risk nonBRCA1/2 French Canadian breast cancer families. Fam Cancer 2007;6:483-490.

6. Nelen MR, Kremer H, Konings IB, et al. Novel PTEN mutations in patients with Cowden disease: absence of clear genotypephenotype correlations. Eur J Human Genet 1999;7:267-273.

7. Lv JW, Cheng TL, Qiu ZL, Zhou WH. Role of the PTEN signaling pathway in autism spectrum disorder. Neurosci Bull 2013;29:773778.

8. Zhou J, Parada LF. PTEN signaling in autism spectrum disorders. Curr Opin Neurobiol 2012;22:873-879.

9. Tan MH, Mester J, Peterson C, et al. A clinical scoring system for selection of patients for PTEN mutation testing is proposed on the basis of a prospective study of 3042 probands. Am J Hum Genet 2011;88:42-56.

10. Chen ZH, Zhu M, Yang J, et al. PTEN interacts with histone $\mathrm{H} 1$ and controls chromatin condensation. Cell Rep 2014;8:2003-2014.

11. Pulido R, Baker SJ, Barata JT, et al. A unified nomenclature and amino acid numbering for human PTEN. Sci Signal 2014;7:1-4.

12. Goffin A, Hoefsloot LH, Bosgoed E, Swillen A, Fryns JP. PTEN mutation in a family with cowden syndrome and autism. Am J Med Genet 2001;105:521-524.

13. Butler MG, Dasouki MJ, Zhou XP, et al. Subset of individuals with autism spectrum disorders and extreme macrocephaly associated with germline PTEN tumour suppressor gene mutations. J Med Genet 2005;42:318-321.

14. Marchese M, BMC Med Genet, 2014.

15. Hobert JA, Embacher R, Mester JL, Frazier TW, 2nd, Eng C. Biochemical screening and PTEN mutation analysis in individuals with autism spectrum disorders and macrocephaly. Eur J Human Genet 2014;22:273-276.

16. Klein S, Sharifi-Hannauer P, Martinez-Agosto JA. Macrocephaly as a clinical indicator of genetic subtypes in autism. Autism Res 2013;6:51-56.

17. McBride KL, Varga EA, Pastore MT, et al. Confirmation study of PTEN mutations among individuals with autism or developmental delays/mental retardation and macrocephaly. Autism Res 2010;3: 137-141.

18. Varga EA, Pastore M, Prior T, Herman GE, McBride KL. The prevalence of PTEN mutations in a clinical pediatric cohort with autism spectrum disorders, developmental delay, and macrocephaly. Genet Med 2009;11:111-117. 
19. Orrico A, Clin Genet, 2009.

20. Herman GE, Butter E, Enrile B, Pastore M, Prior TW, Sommer A. Increasing knowledge of PTEN germline mutations: Two additional patients with autism and macrocephaly. Am J Med Genet A 2007;143:589-593.

21. Buxbaum JD, Cai G, Chaste P, et al. Mutation screening of the PTEN gene in patients with autism spectrum disorders and macrocephaly. Am J Med Genet B Neuropsychiatr Genet 2007;144:484-491.

22. Fombonne E, Roge B, Claverie J, Courty S, Fremolle J. Microcephaly and macrocephaly in autism. J Autism Dev Dis 1999;29:113-119.

23. O'Reilly H, Thiebaut FI, White SJ. Is macrocephaly a neural marker of a local bias in autism? Dev Cogn Neurosci 2013;6:149-154.

24. Bigler ED, Abildskov TJ, Petrie JA, et al. Volumetric and voxelbased morphometry findings in autism subjects with and without macrocephaly. Dev Neuropsychol 2010;35:278-295.

25. Mester JL, Tilot AK, Rybicki LA, Frazier TW, 2nd, Eng C. Analysis of prevalence and degree of macrocephaly in patients with germline PTEN mutations and of brain weight in Pten knock-in murine model. Eur J Human Genet 2011;19:763-768.

26. Frazier TW, Embacher R, Tilot AK, Koenig K, Mester J, Eng C. Molecular and phenotypic abnormalities in individuals with germline heterozygous PTEN mutations and autism. Mol Psychiatry 2014 Oct 7 [Epub ahead of print].

27. Vanderver A, Tonduti D, Kahn I, et al. Characteristic brain magnetic resonance imaging pattern in patients with macrocephaly and PTEN mutations. Am J Med Genet A 2014;164:627-633.

28. Harrington EP, Zhao C, Fancy SP, Kaing S, Franklin RJ, Rowitch DH. Oligodendrocyte PTEN is required for myelin and axonal integrity, not remyelination. Ann Neurol 2010;68:703-716

29. Goebbels S, Oltrogge JH, Wolfer S, et al. Genetic disruption of Pten in a novel mouse model of tomaculous neuropathy. EMBO Mol Med 2012;4:486-499.

30. Cheung KM, Lam CW, Chan YK, Siu WK, Yong L. Atypical focal cortical dysplasia in a patient with Cowden syndrome. Hong Kong Med J 2014;20:165-167.

31. Child ND, Cascino GD. Mystery case: Cowden syndrome presenting with partial epilepsy related to focal cortical dysplasia. Neurology 2013;81:e98-e99.

32. Elia M, Amato C, Bottitta M, et al. An atypical patient with Cowden syndrome and PTEN gene mutation presenting with cortical malformation and focal epilepsy. Brain Dev 2012;34:873-876.

33. O'Rourke DJ, Twomey E, Lynch SA, King MD. Cortical dysplasia associated with the PTEN mutation in Bannayan Riley Ruvalcaba syndrome: a rare finding. Clin Dysmorphol 2012;21:91-92.

34. Conti S, Condo M, Posar A, et al. Phosphatase and tensin homolog (PTEN) gene mutations and autism: literature review and a case report of a patient with Cowden syndrome, autistic disorder, and epilepsy. J Child Neurol 2012;27:392-397.

35. Ngeow J, Stanuch K, Mester JL, Barnholtz-Sloan JS, Eng C. Second malignant neoplasms in patients with Cowden syndrome with underlying germline PTEN mutations. J Clin Oncol 2014;32: 1818-1824.

36. Milas M, Mester J, Metzger R, et al. Should patients with Cowden syndrome undergo prophylactic thyroidectomy? Surgery 2012;152: 1201-1210.

37. Li J, Yen C, Liaw D, et al. PTEN, a putative protein tyrosine phosphatase gene mutated in human brain, breast, and prostate cancer. Science 1997;275:1943-1947.

38. Lee JO, Yang H, Georgescu MM, et al. Crystal structure of the PTEN tumor suppressor: implications for its phosphoinositide phosphatase activity and membrane association. Cell 1999;99: 323-334.

39. Gil A, Andrés-Pons A, Fernández E, et al. Nuclear localization of PTEN by a Ran-dependent mechanism enhances apoptosis:
Involvement of an N-terminal nuclear localization domain and multiple nuclear exclusion motifs. Mol Biol Cell 2006;17:4002-4013.

40. Denning G, Jean-Joseph B, Prince C, Durden DL, Vogt PK. A short $\mathrm{N}$-terminal sequence of PTEN controls cytoplasmic localization and is required for suppression of cell growth. Oncogene 2007;26:3930-3940.

41. Walker SM, Leslie NR, Perera NM, Batty IH, Downes CP. The tumour-suppressor function of PTEN requires an N-terminal lipidbinding motif. Biochem J 2004;379:301-307.

42. Jurado S, Benoist M, Lario A, Knafo S, Petrok CN, Esteban JA. PTEN is recruited to the postsynaptic terminal for NMDA receptordependent long-term depression. EMBO J 2010;29:2827-2840.

43. Hopkins BD, Fine B, Steinbach N, et al. A secreted PTEN phosphatase that enters cells to alter signaling and survival. Science 2013;341:399-402.

44. Liang H, He S, Yang J, et al. PTEN $\alpha$, a PTEN isoform translated through alternative initiation, regulates mitochondrial function and energy metabolism. Cell Metab 2014;19:836-848.

45. He X, Arrotta N, Radhakrishnan D, Wang Y, Romigh T, Eng C. Cowden syndrome-related mutations in PTEN associate with enhanced proteasome activity. Cancer Res 2013;73:3029-3040.

46. Maehama T, Dixon JE. The tumor suppressor, PTEN/MMAC1, dephosphorylates the lipid second messenger, phosphatidylinositol 3,4,5-trisphosphate. J Biol Chem 1998;273:13375-13378.

47. Engelman JA, Luo J, Cantley LC. The evolution of phosphatidylinositol 3-kinases as regulators of growth and metabolism. Nat Rev Genet 2006;7:606-619.

48. Inoki $\mathrm{K}, \mathrm{Li} \mathrm{Y}, \mathrm{Zhu} \mathrm{T}$, Wu J, Guan KL. TSC2 is phosphorylated and inhibited by Akt and suppresses mTOR signalling. Nat Cell Biol 2002;4:648-657.

49. Redfern RE, Daou MC, Li L, Munson M, Gericke A, Ross AH. A mutant form of PTEN linked to autism. Protein Sci 2010;19:19481956.

50. Rodriguez-Escudero I, Oliver MD, Andres-Pons A, Molina M, Cid VJ, Pulido R. A comprehensive functional analysis of PTEN mutations: implications in tumor- and autism-related syndromes. Hum Mol Genet 2011;20:4132-4142.

51. Spinelli L, Black FM, Berg JN, Eickholt BJ, Leslie NR. Functionally distinct groups of inherited PTEN mutations in autism and tumour syndromes. J Med Genet 2015;52:128-134.

52. Kadesjo B, Gillberg C, Hagberg B. Brief report: autism and Asperger syndrome in seven-year-old children: a total population study. J Autism Dev Dis 1999;29:327-331.

53. Newschaffer CJ, Croen LA, Daniels J, et al. The epidemiology of autism spectrum disorders. Annu Rev Public Health 2007;28:235258.

54. Sponheim E, Skjeldal O. Autism and related disorders: Epidemiological findings in a Norwegian study using ICD-10 diagnostic criteria. J Autism Dev Dis 1998;28:217-227.

55. Wingate M, Kirby RS, Pettygrove S, et al. Prevalence of autism spectrum disorder among children aged 8 years-Autism and Developmental Disabilities Monitoring Network, 11 Sites, United States, 2010. MMWR Surveill Summ 2014;63:1-21.

56. Minaguchi T, Waite KA, Eng C. Nuclear localization of PTEN is regulated by $\mathrm{Ca}(2+)$ through a tyrosil phosphorylation-independent conformational modification in major vault protein. Cancer Res 2006;66:11677-11682.

57. Trotman LC, Wang X, Alimonti A, et al. Ubiquitination regulates PTEN Nuclear import and tumor suppression. Cell 2007;128:141156.

58. Lobo GP, Waite KA, Planchon SM, Romigh T, Houghton JA, Eng C. ATP modulates PTEN subcellular localization in multiple cancer cell lines. Hum Mol Genet 2008;17:2877-2885.

59. Chung JH, Eng C. Nuclear-cytoplasmic partitioning of phosphatase and tensin homologue deleted on chromosome 10 (PTEN) 
differentially regulates the cell cycle and apoptosis. Cancer Res 2005;65:8096-8100.

60. Shen WH, Balajee AS, Wang J, et al. Essential role for nuclear PTEN in maintaining chromosomal integrity. Cell 2007;128:157170.

61. Tilot AK, Gaugler MK, Yu Q, et al. Germline disruption of Pten localization causes enhanced sex-dependent social motivation and increased glial production. Hum Mol Genet 2014;23:3212-3227.

62. Kwon CH, Luikart BW, Powell CM, et al. Pten regulates neuronal arborization and social interaction in mice. Neuron 2006;50:377388.

63. Wang Y, Cheng A, Mattson MP. The PTEN Phosphatase is essential for long-term depression of hippocampal synapses. Neuromolecular Med 2006;8:329-336.

64. Sperow M, Berry RB, Bayazitov IT, Zhu G, Baker SJ, Zakharenko SS. Phosphatase and tensin homologue (PTEN) regulates synaptic plasticity independently of its effect on neuronal morphology and migration. J Physiol 2012;590:777-792.

65. Takeuchi K, Gertner MJ, Zhou J, Parada LF, Bennett MV, Zukin RS. Dysregulation of synaptic plasticity precedes appearance of morphological defects in a Pten conditional knockout mouse model of autism. Proc Natl Acad Sci U S A 2013;110:4738-4743.

66. Di Cristofano A, Kotsi P, Peng YF, Cordon-Cardo C, Elkon KB, Pandolfi PP. Impaired fas response and autoimmunity in Pten $^{+/}$ mice. Science 1999;285:2122-2125.

67. Amiri A, Cho W, Zhou J, et al. Pten deletion in adult hippocampal neural stem/progenitor cells causes cellular abnormalities and alters neurogenesis. J Neurosci 2012;32:5880-5890.

68. Kazdoba TM, Sunnen CN, Crowell B, Lee GH, Anderson AE, D'Arcangelo G. Development and characterization of NEX- Pten, a novel forebrain excitatory neuron-specific knockout mouse. Dev Neurosci 2012;34:198-209.

69. Voineagu I, Wang X, Johnston P, et al. Transcriptomic analysis of autistic brain reveals convergent molecular pathology. Nature 2011;474:380-384.

70. Courchesne E. Brain development in autism: early overgrowth followed by premature arrest of growth. Ment Retard Dev Disabil Res Rev 2004;10:106-111.

71. Fraser MM, Zhu X, Kwon C-HH, Uhlmann EJ, Gutmann DH, Baker SJ. Pten loss causes hypertrophy and increased proliferation of astrocytes in vivo. Cancer Res 2004;64:7773-7779.

72. Zhou J, Blundell J, Ogawa S, et al. Pharmacological inhibition of mTORC1 suppresses anatomical, cellular, and behavioral abnormalities in neural-specific Pten knock-out mice. J Neurosci 2009;29:1773-1783.

73. Kwon CH, Zhu X, Zhang J, et al. Pten regulates neuronal soma size: a mouse model of Lhermitte-Duclos disease. Nat Genet 2001;29: 404-411.

74. Fraser MM, Bayazitov IT, Zakharenko SS, Baker SJ. Phosphatase and tensin homolog, deleted on chromosome 10 deficiency in brain causes defects in synaptic structure, transmission and plasticity, and myelination abnormalities. Neuroscience 2008;151:476-488.
75. Luikart BW, Schnell E, Washburn EK, Bensen AL, Tovar KR, Westbrook GL. Pten knockdown in vivo increases excitatory drive onto dentate granule cells. J Neurosci 2011;31:4345-4354.

76. Haws ME, Jaramillo TC, Espinosa F, et al. PTEN knockdown alters dendritic spine/protrusion morphology, not density. J Comp Neurol 2014;522:1171-1190.

77. Flores AI, Narayanan SP, Morse EN, et al. Constitutively active Akt induces enhanced myelination in the CNS. J Neurosci 2008;28: 7174-7183.

78. Maximo JO, Cadena EJ, Kana RK. The implications of brain connectivity in the neuropsychology of autism. Neuropsychol Rev 2014;24:16-31.

79. Peradones C, Costanzo RV, Kowaljow V, Pivetta OH, Carminatti H, Radrizzani M. Correlation between synaptogenesis and the PTEN phosphatase expression in dendrites during postnatal brain development. Brain Res Mol Brain Res 2004;128:8-19.

80. Arendt KL, Benoist M, Lario A, Draffin JE, Munoz M, Esteban JA. PTEN counteracts PIP3 upregulation in spines during NMDAreceptor-dependent long-term depression. J Cell Sci 2014;127: 5253-5260.

81. Briz V, Hsu YT, Li Y, Lee E, Bi X, Baudry M. Calpain-2-mediated PTEN degradation contributes to BDNF-induced stimulation of dendritic protein synthesis. J Neurosci 2013;33:4317-4328.

82. Ventruti A, Kazdoba TM, Niu S, D'Arcangelo G. Reelin deficiency causes specific defects in the molecular composition of the synapses in the adult brain. Neuroscience 2011;189:32-42.

83. Xiong Q, Oviedo HV, Trotman LC, Zador AM. PTEN regulation of local and long-range connections in mouse auditory cortex. $\mathrm{J}$ Neurosci 2012;32:1643-1652.

84. Clipperton-Allen AE, Page DT. Pten haploinsufficient mice show broad brain overgrowth but selective impairments in autismrelevant behavioral tests. Hum Mol Genet 2014;23:3490-3505.

85. Page DT, Kuti OJ, Prestia C, Sur M. Haploinsufficiency for Pten and serotonin transporter cooperatively influences brain size and social behavior. Proc Natl Acad Sci U S A 2009;106:1989-1994.

86. Ogawa S, Kwon C-H, Zhou J, Koovakkattu D, Parada LF, Sinton C. A seizure-prone phenotype is associated with altered freerunning rhythm in Pten mutant mice. Brain Res 2007;1168:112123.

87. Lugo JN, Smith GD, Arbuckle EP, et al. Deletion of PTEN produces autism-like behavioral deficits and alterations in synaptic proteins. Front Mol Neurosci 2014;7:27.

88. Clipperton-Allen AE, Page DT. Decreased aggression and increased repetitive behavior in Pten haploinsufficient mice. Genes Brain Behav 2015;14:145-157.

89. Bonde E. Comorbidity and subgroups in childhood autism. Eur Child Adolesc Psychiatry 2000;9:7-10.

90. Pal A, Barber TM, Van de Bunt M, et al. PTEN mutations as a cause of constitutive insulin sensitivity and obesity. N Engl J Med 2012;367:1002-1011. 